\title{
MULTI-AGENT SIMULATION OF ALLOCATING AND ROUTING AMBULANCES UNDER CONDITION OF STREET BLOCKAGE AFTER NATURAL DISASTER
}

\author{
S. Azimi a, M. R. Delavar ${ }^{b}$, A. Rajabifard ${ }^{\mathrm{c}}$
}

\begin{abstract}
${ }^{a}$ GIS Dept., School of Surveying and Geospatial Eng., College of Eng., University of Tehran, Tehran, Iran- sanazazimi@ut.ac.ir
${ }^{\mathrm{b}}$ Center of Excellence in Geomatic Eng. in Disaster Management, School of Surveying and Geospatial Eng., College of Eng., University of Tehran, Tehran, Iran-mdelavar@ut.ac.ir

${ }^{c}$ Department of Infrastructure Engineering, Centre for Spatial Data Infrastructures and Land Administration, The University of Melbourne, Melbourne, VIC, Australia-abbas.r@unimelb.edu.au
\end{abstract}

\section{Commission IV, WG IV/3}

KEY WORDS: Allocation, Constraint Voronoi diagram, Simulated annealing, NSGA $\Pi$, Smart government

\begin{abstract}
:
In response to natural disasters, efficient planning for optimum allocation of the medical assistance to wounded as fast as possible and wayfinding of first responders immediately to minimize the risk of natural disasters are of prime importance. This paper aims to propose a multi-agent based modeling for optimum allocation of space to emergency centers according to the population, street network and number of ambulances in emergency centers by constraint network Voronoi diagrams, wayfinding of ambulances from emergency centers to the wounded locations and return based on the minimum ambulances travel time and path length implemented by NSGA $\boldsymbol{\Pi}$ and the use of smart city facilities to accelerate the rescue operation. Simulated annealing algorithm has been used for minimizing the difference between demands and supplies of the constrained network Voronoi diagrams. In the proposed multi-agent system, after delivering the location of the wounded and their symptoms, the constraint network Voronoi diagram for each emergency center is determined. This process was performed simultaneously for the multi-injuries in different Voronoi diagrams. In the proposed multiagent system, the priority of the injuries for receiving medical assistance and facilities of the smart city for reporting the blocked streets was considered. Tehran Municipality District 5 was considered as the study area and during 3 minutes intervals, the volunteers reported the blocked street. The difference between the supply and the demand divided to the supply in each Voronoi diagram decreased to 0.1601. In the proposed multi-agent system, the response time of the ambulances is decreased about $36.7 \%$.
\end{abstract}




\section{INTRODUCTION}

Natural disasters cause large scale loss of life, damage society's infrastructure, housing, and industrial complex. For responding to natural disasters, efficient planning is very important and allocation of efficient emergency response facilities as fast as possible is considered ( $\mathrm{Li}$ et al.,2011). The response time of the first responders to disasters for rescuing the wounded is a critical factor that affects life care. The way to allocate the emergency centers to the wounded is affected by the delivering time of ambulances to the wounded location. Therefore, the optimum allocation of ambulances to the injured people as fast as possible is necessary where selecting an optimum route for accessing the ambulances to the injuries locations require medical aid at the same time.

Search and rescue operation is a good application for agent-based social simulations (ABSS). Agent based models assist studying a number of aspects of dynamic environments which is very similar to real life systems (Chavoshi et al., 2008).

There is no common agreement about the accurate definition of the term 'agent', although definitions agree on more points than they disagree (Macal and North, 2005). An agent perceives its environment through sensors and acts upon it through its effectors (Rusell and Norvig, 2003). An agent is as an autonomous and proactive system that is capable of interacting with other agents to satisfy its objectives and learn from its environment and change its behaviors, accordingly. Agent-based models consist of multiple agents, interacting with each other, situated within a model or simulation environment. A multiagent system consists of several agents interacting with each other and with objects in a single environment, for achieving a universal goal (Roozemond, 2001).

There are a number of studies on optimizing the allocation of the wounded to hospitals, wayfinding of the emergency vehicles to the injuries location and transferring them to the emergency centers and multi-agent simulation of the rescue operation.

Voronoi diagrams are used for facility allocation. Karimi (2009) considered Adaptive Multiplicatively Weighted Network Voronoi Diagram (AMWNVD) for the allocation of the injured people to emergency centers in order to reduce the delay of the ambulances' dispatching. In the MWNVDs, the weight of each emergency center is determined by the number of the ambulances in each center. Also, the blocks population and urban street were used for allocation of the Voronoi diagrams to the emergency centers. In the AMWNVD, the MWNVD was solved repeatedly with linear optimization of weights of emergency centers, the difference between supply and demand was decreased and the result was closer to the optimum (Karimi, 2009). Chavoshi et al. (2008) modeled optimum agent based strategies for wayfinding for the first responders based on landmarks remained after the earthquake in a multi-agent system. Also, the effects of blocked roads and collapsed buildings were considered in this model. The proposed simulation model focuses on information exchange between agents and the landmarks. The extracted buildings from the risk map were considered as the landmarks to guide the rescue operation to the desired destination. Finally, optimum route for the first responder based on special condition after the earthquake was determined (Chavoshi et al., 2008). Wang and Zlatanova (2016) proposed a multi-agent system for navigating responders to destinations to respond to a disaster when the disaster causes different kinds of moving obstacles such as fires, plumes and floods. The proposed multi-agent based navigation system consists of information collection from hazard simulations, geodatabase development, monitoring rescue operation, path planning and visualization modules. The geodatabase and visualization modules support the components for the proposed multi-agent system. The monitoring module has task monitoring, network monitoring and vehicle monitoring agents. The path planning module plans the route and send this to the vehicle agents. The determination of optimum route is done in the path planning module (Wang and Zlatanova, 2016).

The condition of street networks after disasters occurrence is completely different. Natural disasters damage to infrastructures, because of the possibility of buildings collapse, may lead to traffic in the street networks which results some parts of the road network is temporarily unavailable. Because of the blockage of many streets after the disasters, analysis of the accessibility of street networks is of prime importance.

In addition to the historical travel time of street networks acquired from some official organizations, the existence of near real time data of traffic volume of the vehicles and the blockage of the streets for routing emergency vehicles is noticeable.

In traditional disaster management systems, citizens have been considered as people to be rescued. However, the disaster management of smart city can benefit from citizen participation for providing information about fire location, street blockage, collapsed buildings and the injured people location (Foresti et al., 2015).

There are a number of studies on disaster management in the smart city. Hirokawa and Osaragi (2016) have analyzed the accessibility of firefighters to the location of fire immediately after an earthquake. A* algorithm is used for finding the fastest path on the street network by the firefighters. When volunteers collected information on street blockage, the access time of the firefighters to the location of fire have decreased in the same level as the situation that no street was blocked (Hirokawa and Osaragi, 2016).

In this paper, we are faced with the question that, how to expedite the allocation of ambulances to the wounded and wayfinding the ambulances in a rescue operation after natural disasters, based on the facilities in a smart city and modeling of the multi-agent system?

In this paper, the multi-agent rescue operation that integrated the allocation of the ambulances to the wounded locations, the wayfinding of the ambulances and the facilities of the smart city to accelerate the rescue operation after a natural disaster, have been proposed. In this multi-agent system, emergency centers, streets, injuries, ambulances, volunteers and their rescue schedule are modeled as agents in Anylogic simulation software, having their own attributes and behaviors.

The hypothesis of this paper is that optimum allocation of the ambulances to the injuries' locations according to the priorities of the injuries and wayfinding the ambulances based on the minimum ambulances travel time and path length due to the specified blocked streets reported by the volunteers could accelerate the rescue operation.

In the proposed multi-agent rescue operation, optimum allocation of the ambulances from the emergency center to the injuries' location was performed by constraint network Voronoi diagrams. It is assumed that the injured can send their locations by mobile Global Positioning System (GPS) and their initial symptoms to emergency centers. In addition, it is assumed that the delivered ambulances from one emergency center, transfer the wounded to the same emergency center. The minimum travel time from the fire station to the blocked street is assumed to be the blockage time of these streets.

In the proposed multi-agent rescue operation, the location of the wounded agents and the symptom of the injured are sent to the emergency centers. After determining the Voronoi diagram of the wounded persons, the located emergency center in this Voronoi diagram, dispatched an ambulance to the wounded 
person location. The non-dominated sorting genetic algorithm $\Pi$ (NSGA $\Pi$ ) (Deb et al., 2002) is used for finding the optimal path of the dispatched ambulances. This process was performed simultaneously for the injured in different Voronoi diagrams. Priorities of the injured for the dispatching ambulances in each Voronoi diagrams is determined by the severity of the injured victims. Because of the street blockage after the natural disaster, local residents reported near real time information about blocked streets. So, the multi-agent rescue operation simulation is updated by the reported massages and then the wayfinding algorithm performed again. The proposed multi-agent rescue operation can decrease the gap between demand and supply in each emergency center Voronoi diagram and the ambulances' delay time for allocation of the medical assistance to the injuries. The rest of this paper is organized as follows: Section 2 explains the methodology of the proposed multi-agent rescue operation model consisting of the allocation of the injuries to hospitals and the wayfinding of the ambulances in the disaster situation. Section 3 explains implementation and results of the proposed multi-agent rescue operation. Finally, Section 4 presents conclusion and directions for future research.

\section{METHODOLOGY}

The proposed multi-agent rescue operation system consists of the optimum allocation of the ambulances to the wounded locations, the optimum routing of the ambulances in the rescue operation and the smart city issues. In the following, each of the topics is described separately.

\subsection{Allocation}

Space allocation of urban facilities divides a territory into subareas based on specific criteria. The voronoi diagram can be used to specify service area of each facility. In this study, constrained Voronoi diagrams was used for space allocation of urban emergency centers according to population, supply and street network. The number of ambulances in each emergency center is considered as an emergency center weight. The iterative mechanism in the Multiplicatively Weighted Network Voronoi Diagram (MWNVD) with correcting emergency center weights in each iteration, decreases the difference between supply (the expected population lives in the region for using the services) and demand (the population lives in the region). The simulated annealing optimization technique is used for minimizing the difference between supply and demand in each Voronoi diagram. After the disaster happened, the injuries send their locations and initial symptoms to the emergency centers. Then the Voronoi diagram contained the location of demand center is specified. So, the emergency center is assigned to this demand center. The emergency center received this demand and delivered the ambulance to the demand location. This process is performed for multi demands in different Voronoi diagrams simultaneously. The noticeable point during the allocation process was the priority for each demand. The emergency center assigned the priority to these injuries based on their delivered symptoms. The severity of injury determined the priority of the demands. The wounded with injuries more quickly receive medical assistance. This analysis is performed for each Voronoi diagrams, separately.

In the following, the AMWNVD and simulated annealing techniques will be described in detail.

\subsubsection{Voronoi diagram}

A Voronoi diagram (Aurenhammer, 1991) represents the partition of the given space into regions, with the bounds determined by distances to a specified family of objects. In the ordinary Voronoi diagram, all generators start growing at identical rates, simultaneously. In the ordinary Voronoi diagram, Euclidian distance is used to define service area of each facility. Because of the defects and limitations of the standard Voronoi diagram for allocating space to services' centers, MWVD (Reitsma et al., 2004) was employed. In MWVD, the weight of each region is different. In the other word, all generators start growing at different rate simultaneously. The rates of generators determined by their weights. Eq (1) shows Multiplicatively weighted distance for determining the bound of each region in MWVD and MWVD is shown as Eq (2) (Okabe et al., 2000).

$$
\begin{gathered}
d_{M W}=\frac{1}{w_{i}} \times\left|p-p_{i}\right| \\
\left(p_{i}\right)=\left\{p\left|\in R^{2}\right| \frac{1}{w_{i}}|| p-p_{i}|| \leq \frac{1}{w_{j}}|| p-p_{j}||,\right. \\
j \neq i, i=1, \ldots, n\}
\end{gathered}
$$

where $w=\left\{w_{1}, \ldots, w_{n}\right\}$ is the related weight to generator points $P=\left\{p_{1}, \ldots, p_{n}\right\}$ $p \in R^{2}$

In AMWVD, the MWVD has solved repeatedly that the weight of each new solution is dependent on the previous solution and is determined by an error of the previous solution. Eq (3) shows the relation of the amount of the provided services and the weight of services centers (Reitsma et al., 2004, 2007).

$$
\frac{w_{k}}{w_{j}}=\frac{q_{k}}{q_{j}}
$$

where $q=$ the amount of services in services' centers (the population requiring facilities and services)

In this study, Voronoi diagrams are based on the street network. For determining the boundary of each region, the distance on the street network which is different from Euclidean distance was evaluated.

Network $N$ consists of a set of nodes $\left(N=\left\{p_{1}, \ldots, p_{l}\right\}\right)$ and links $\left(L=\left\{l_{1}, \ldots, l_{k}\right\}\right)$ that is shown as $N=(N, l)$. The network distance is defined as the shortest path between one node and one point on the link. In the network Voronoi diagram, at first, the point $p$ in $R^{2}$ space allocated to the nearest point of the link and then this point is allocated to the nearest generator node of the network $\left(p_{i}\right)$ based on the network distance (Karimi, 2009). $d_{a c c}(p, a(p))$ is the shortest distance between $p$ and the nearest point of the link and the shortest network distance between the nearest point of the link and $p_{i}$ is $d_{n e t}\left(p_{i}, a(p)\right)$. Eq (4) shows the distance between $p$ and $p_{i}$. Eq (5) defines the Voronoi diagram of the network (Graf and Winter, 2003).

$$
\begin{gathered}
d_{\text {acc,net }}\left(p, p_{i}\right)=d_{a c c}(p, a(p))+d_{n e t}\left(a(p), p_{i}\right) \\
v_{\text {area }}\left(p_{i}\right)=\left\{p \mid d_{\text {acc,net }}\left(p, p_{i}\right) \leq d_{a c c, n e t}\left(p, p_{j}\right), j\right. \\
\left.\in I_{n} \backslash\{i\}\right\}
\end{gathered}
$$

In the MWNVD, the generator points (or service centers) have their specific weight. The Multiplicatively Weighted distance is shown using Eq (6) (Okabe et al., 2008):

$$
d_{M W N}\left(p, p_{i}\right)=B_{i} d_{n e t}\left(p, p_{i}\right)
$$

where $\quad \mathrm{a}_{i}=$ the weight of $p_{i}$ $B_{i}=\frac{1}{\mathrm{a}_{i}}$ 


\subsubsection{Simulated Annealing}

Simulated Annealing (SA) (Yang, 2010) is a probabilistic metaheuristic technique for the optimization problem. The annealing refers to the fast heating of a metal and then cooling it gradually. If enough randomness is used with very slow cooling, the SA can find the global minimum. The SA algorithm is to use random search which not only accepts changes that improve the objective function, but also keeps some changes with probability $\mathrm{p}$, called transition probability, that do not improve the objective function. Eq (7) shows the probability $p$ (Yang, 2010).

$$
p(\Delta f, T)=e^{\frac{-\Delta f}{T}}
$$

A random number ( $\mathrm{r}$ ) is used as a threshold and if $p>r$ is true, a change is accepted. In this study, the geometric schedule to decrease the temperature was considered. As shown in Eq (8), Geometric cooling schedule decreases the temperature essentially by a cooling factor $0<a<1 \quad(a=0.7 \sim 0.95$ is commonly used) (Yang, 2010).

$$
\begin{aligned}
& T(t)=T_{0} \mathrm{a}^{t} \\
& t=1,2, \ldots, t_{f}
\end{aligned}
$$

where $T_{0}=$ initial temperature

In this paper, the SA optimization algorithm was used for the optimizing AMWNVD to assign space to the emergency centers. The SA process continues until the gap between supply and demand is filled and this difference was closer to the optimum. Objective function $f$ is defined as $\mathrm{Eq}(9)$. Eq (10) shows the error metric used in this paper (Reitsma et al., 2007).

$$
\begin{aligned}
& f(i, j)=P_{i}-p_{i, j} \\
& \text { Error }=\frac{P_{i}-p_{i, j}}{P_{i}}
\end{aligned}
$$

where $\quad p_{i, j}=$ the amount of demand in emergency center $j$ in iteration $i$

$P_{j}=$ the amount of expected supply in emergency center $j$ in iteration $i$

Both $p_{i, j}$ and $P_{j}$ are proportions. In AMWNVD, the services' centers weigths is updated in each iteration by the SA until the notion of a difference $f(i, j)$ gets minimized. Eq (11) shows the weight of each emergency center for each iteration (Reitsma et al., 2004, 2007).

$$
\begin{array}{r}
w_{i+1, j}=w_{i, j}+\Delta w \\
i \geq 0.1 \leq j \leq N
\end{array}
$$

where $w_{i, j}=$ weight of generator $j$ in iteration $i$ $w_{i+1, j}=$ weight of generator $j$ in iteration $i+1$ $N=$ the number of generators

\subsection{Routing}

Selection of an optimum route to deliver the ambulance from emergency center to the injury location and transfer the injury to these emergency centers is a very sensitive job. This optimum path is satisfied by two objective functions, including the ambulance travel time and route length. A genetic algorithm was used for determining optimum route for the ambulances from the emergency centers to the location of the injuries and vice versa.
The Genetic algorithm can be used in a single objective vehicle routing problem. However, a multi-objective routing algorithm is required for this study. So, NSGA $\Pi$ (Deb et al., 2002) was considered for the multi-objective routing problem in order to minimize the ambulances travel time and the route length. In this process, the local residents reported blocked streets every 3 minutes. So, optimal routes of ambulances are updated by NSGA $\boldsymbol{\Pi}$. This process is performed repeatedly until all the injuries are transferred to the emergency centers.

\subsubsection{Genetic algorithm}

Genetic algorithms (Mitchell, 1998) was invented by John Holland in 1960 as a useful tool for solving routing search and optimization problems. Genetic algorithm represents a random search for solving optimization problems.

The genetic algorithm always considers a population of solutions and each solution is encoded as arrays of character strings to represent a chromosome-like data structure. Generation is one iteration of creating a new population. Three central operators of the simplest genetic algorithm are selection, crossover (single point), and mutation (Yang, 2010). The selection operator selects the pair of parent chromosome of the current generation for reproduction. The chromosomes with a high fitness, have a high chance for selection. There are a number of ways for the random selection such as roulette wheel selection and tournament selection. The Crossover operator selects "parents" from the current population of the chromosomes for generating the next generation. This operator has a probability for crossover randomly paired chromosomes. The Mutation operator shows the string deliberately changed to maintaining diversity in the population. There was mutate probability for each bit in the population, which is usually very small (e.g., 0.001) (Yang, 2010).

A random population is generated as the first step in a genetic algorithm. Then the fitness of each chromosome in the current generation is evaluated by a proper fitness function. Then, the selection, crossover and mutation operators are performed for creating a new generation. This cycle is repeated for a number of generations until termination criteria are met.

\subsubsection{NSGA}

Generally, the concept of routing optimization for a multiobjective problem is different from the single-objective optimization problem. The multi-objective shortest path problem finds a set of proper paths with the optimized objectives. Multiobjective shortest path problem needs a set of optimum solutions named Pareto-optimal solutions. The evolutionary algorithms can be used to find multiple Pareto-optimal solutions in a single simulation run.

One of the first such evolutionary algorithms is the nondomination based genetic algorithm (NSGA). NSGA (Srinivas and Deb, 1994) is an effective non-dominated based genetic algorithm for the multi-objective optimization problem. This algorithm has a computational complexity problem and other disadvantages. Because of the disadvantages of this algorithm, an improved version of NSGA, so called NSGA $\boldsymbol{\Pi}$ was proposed (Deb et al., 2002) and (Seshadri, 2007). This algorithm uses the elitist principle and emphasizes on non-dominated solutions. In NSGA $\boldsymbol{\Pi}$, after creating the first generation, each chromosome of the first generation compared with another chromosome to specify if it is dominated. So, individual of the first generation is sorted based on the non-dominated process into each front. A fitness value has been assigned to each individual in each front. These fitness values are determined by the front of each chromosome. The non-dominated individuals in the population being the first front and fitness value of 1 is assigned to them. Individuals who are dominated by the first front population, 
being the second front and fitness value of 2 is assigned to them. This process is repeated to find higher level non-domination. Then, crowding distance parameter is computed for each individual. This parameter determines the Euclidian distance between each individual and its neighbors in the one front based on their multi-objectives. After sorting the set of solutions in one front for every objective function, a large distance (e.g. $\infty$ ) is assigned to boundary solutions and a distance for other solutions are calculated using Eq (12) (Deb et al., 2002).

$$
\begin{aligned}
& d_{i}=d_{i}+\frac{l_{m}^{i+1}-l_{m}^{i-1}}{f_{m}^{\max }-f_{m}^{\min }} \\
& i=2 \text { to } k-1
\end{aligned}
$$

where $\quad l=$ a non-dominated set of solutions in a front $l_{m}^{i}=m_{t h}$ objective function value of $i_{t h}$ individual in the set $l$

$k=$ the number of solution the set $l$ $f_{m}^{\max }=$ maximum value of $m_{t h}$ objective function $f_{m}^{\text {min }}=$ minimum value of $m_{t h}$ objective function

In this paper, the binary tournament selection is considered in the NSGA $\boldsymbol{\Pi}$ multi-objective routing. In the binary tournament selection, the two chromosomes of the current generation were selected, randomly. If these two chromosomes have different ranks, the chromosome having lower rank is selected. However, if they have equal rank, a chromosome with large average crowding distance is selected. The lower front value and the higher average crowding distance make the better diversity in the next generation. The process of binary selection until completion of the pool is continued. The pool size in this study is considered as the size of the initial population. The single point crossover is considered in this paper. Parents for single point crossover is selected from the pool. In the single point crossover, the parts of the two parents after their common route in them (crossover position) are exchanged to form the two offsprings. Also, no mutation is considered. After the single point crossover, the current population and the generated offsprings are sorted based on non-domination and individuals, in the size of the population selected for generating the next generation. This process is repeated until termination criteria become true.

\subsection{Smart city and smart government}

In traditional disaster management systems, citizens have been considered as people to be rescued. In addition to acquiring data from some official organizations, ordinary residents' participation for providing information about affected area is noticeable (Horita et al. 2013). Crowdsourcing has considered in recent years as a significant source of information that can support disaster management in the smart city.

The smart city as an innovative city that using new technologies to connects information, people and city elements in order to improve citizens' quality of life (Bakici et al., 2013). For developing the smart cities, the development of efficient and effective government structure is necessary. The smart governance improves transparency in government, providing public participation in decision making, providing information to the citizen, increasing collaborative activities and so on.

Natural disasters make some parts of the road network temporarily unavailable. The disaster management of the smart city can benefit from citizen participation for providing near real time information about fire locations, blocked streets, collapsed buildings and so on for improving response to natural disaster. The people involved in a disaster area as sensors can provide real time information about the disasters (Poblet et al., 2017). Because of the blockage of many streets after the disasters, analysis of the accessibility of street networks is of prime importance. In this paper, the collected information on street blockages by local residents after a natural disaster is considered. The local residents located at the nearest intersection to their position and reported the blocked streets during 3 minutes intervals. So, the ambulance optimal route consists of at least one of the reported block streets, updated.

\section{IMPLEMENTATION}

The proposed methodology was implemented in a part of municipal District 5 of Tehran. Figure 1 represents the scope of the study area in Open Street Map ${ }^{(1)}$ that is limited to the Shahid Fahmideh highway from North, the Sanaye Hapeymayi street from West, the Lashkari highway from South and the Jenah highway from East. The topographic map of National Cartographic Center (NCC) at the scale of 1:2000 in the shapefile format updated in 2003 and census data extracted from the demographic census in the shapefile format at the scale of 1:2000 carried out in 2007 by Statistical Centre of Iran were used in this study. The locational information of emergency centers was derived from these shapefiles.

As shown in Figure 1, two emergency centers $\mathrm{EC}_{1}$ and $\mathrm{EC}_{2}$ were considered in this study area with Latitude and Longitude of $35^{\circ}$ $42^{\prime} 0.54^{\prime \prime}, 51^{\circ} 19^{\prime} 57.68^{\prime \prime}, 35^{\circ} 42^{\prime} 55.8^{\prime \prime}$ and $51^{\circ} 18^{\prime} 13.68^{\prime \prime}$, respectively. Also, four and five ambulances located in the $\mathrm{EC}_{1}$ and $\mathrm{EC}_{2}$ respectively. In the study area, the fire station is located in $35^{\circ} 42^{\prime} 48.14 " L a t i t u d e$ and $51^{\circ} 18^{\prime} 35.34$ "Longitude. The minimum travel time between the blocked streets and the fire station is considered as the blockage time of these streets.

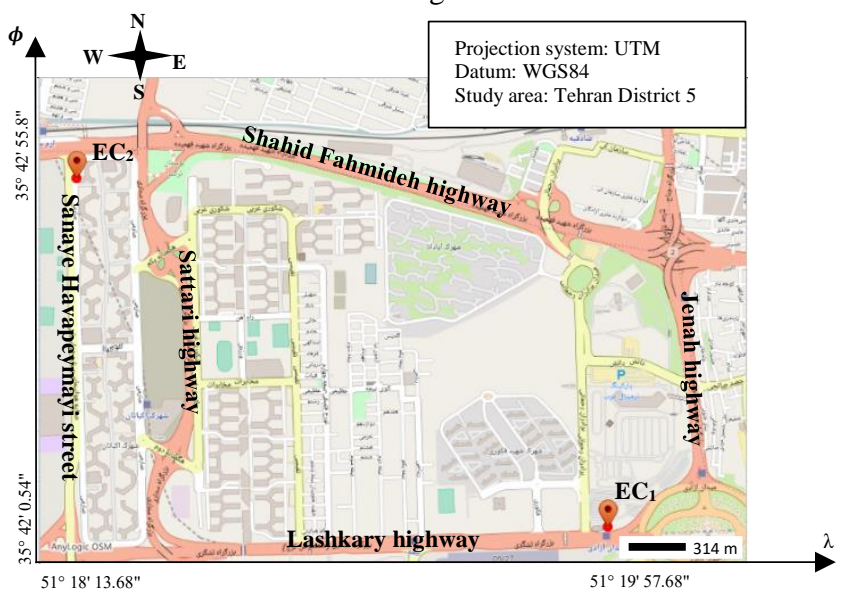

Figure 1. The study area (www.openstreetmap.org)

The population of each block is determined by the proportion of the area of the block to the area of the statistical zone. For the implementation of AMWNVD, the central axis of the urban street network was drawn in AutoCAD map software. Also, the historical travel time data of urban street network achieved from Municipality of Tehran Transport and Traffic company. The travel time data are collected in 2017 in the shape file format. As mentioned, travel time and length of streets were used in finding the ambulance optimum path. The street network of the Municipal District 5 of Tehran is obtained from Municipality of Tehran Control Traffic Company.

Minimization of the gap between demand and supply of the constrained network Voronoi diagrams implemented by SA. The SA optimization process of AMWNVD is shown in Figure 2,

\footnotetext{
(1) Www.openstreetmap.org
} 
where $\mathrm{f}$ is the objective function, $T_{0}$ is the initial temperature, $x$ is the initial solution, $N_{\max }$ is the maximum number of generation and $T_{f}$ is the final temperature. Dijkstra routing algorithm was used for determining the distance of each node of the street network to the emergency centers on the street network in constraint Voronoi diagrams. The number of ambulances in each emergency center considered as emergency center weight. An SA optimization was done in 40 iterations in MATLAB where $a=0.7$. The Error was obtained equal to 0.1601 by Eq (10). Figures 3 shows final AMWNVDs by SA in ArcMap. The location of the emergency centers in red and center of blocks in green are presented. Also, the yellow streets of the street network are assigned to $\mathrm{EC}_{1}$ and the purple streets are assigned to $\mathrm{EC}_{2}$.

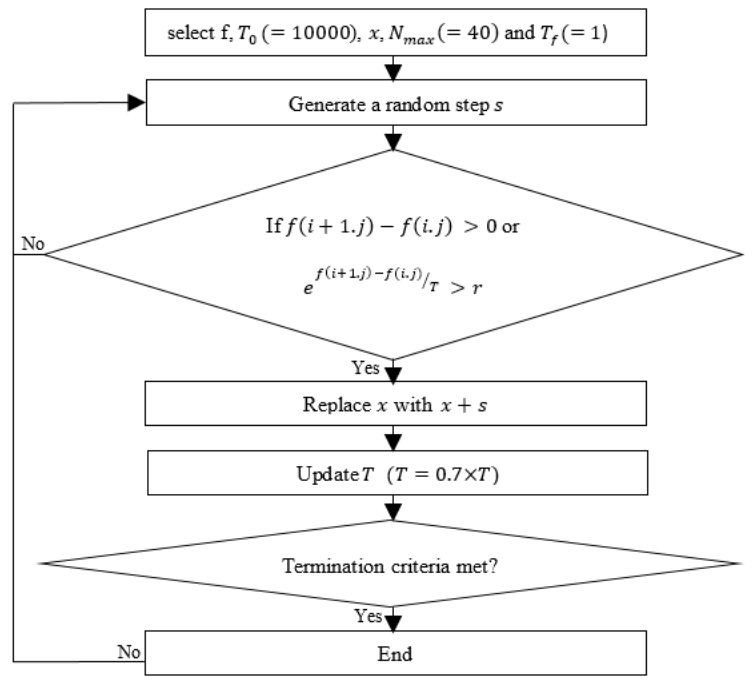

Figure 2. The flowchart of SA optimization of AMWNVD

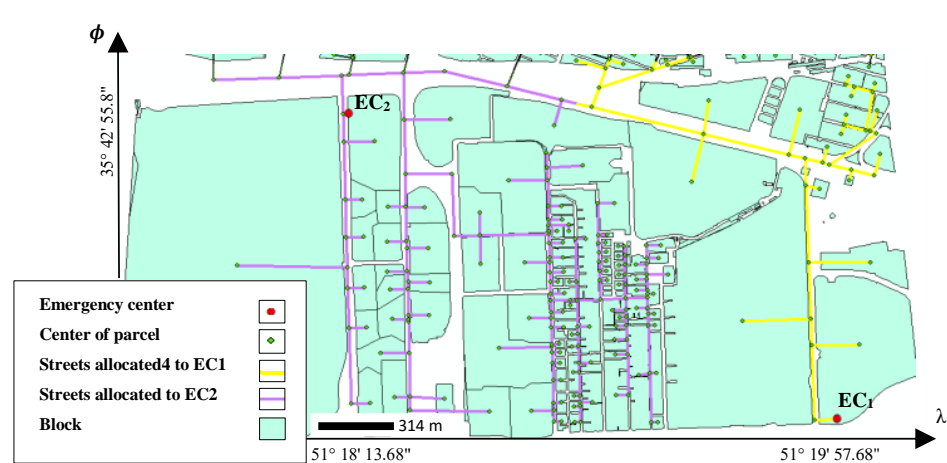

Figure 3. The SA optimization results of AMWNVDs for the emergency centers in ArcMap

The multi-objective NSGA $\boldsymbol{\Pi}$ algorithm is used for wayfinding the ambulances in the rescue operation. This multi-objective algorithm determines the optimal route according to ambulances travel time and length of ambulance route from the emergency center to the demand center and vice versa. In this study, strings of random routes from the origin to the destination are considered as chromosomes for forming the initial population of NSGA $\boldsymbol{\Pi}$. The size of the initial population based on the total number of routes from the origin to the destination is variable. The maximum initial population size and generation number are considered as 10 and 12, respectively. As mentioned, the sum of the distances traversed in each route and sum of travel times of each segment based on the historical travel time data were considered as the fitness functions. The optimum solution is

\footnotetext{
(2) Www.anylogic.com
}

related to the shorter distance and travel time. The crossover rate was selected as 0.9 . The route with the minimum travel time and length is selected as ambulance optimum path. Figure 4 shows the flowchart of NSGA $\boldsymbol{\Pi}$.

The proposed multi-agent rescue operation model was implemented in Any logic software consisting of emergency centers, streets, injuries, ambulances, volunteers and their rescue schedule agents. AnyLogic (2) is a proprietary multi-method simulation software consisting of system dynamics, processcentric and agent based modeling. This simulation software is a native Java environment. Anylogic has a traffic library for simulating vehicle congestion on highways and streets, parking lots and so on. The Anylogic simulation language consists of Statecharts that are mostly used for defining agent behavior.

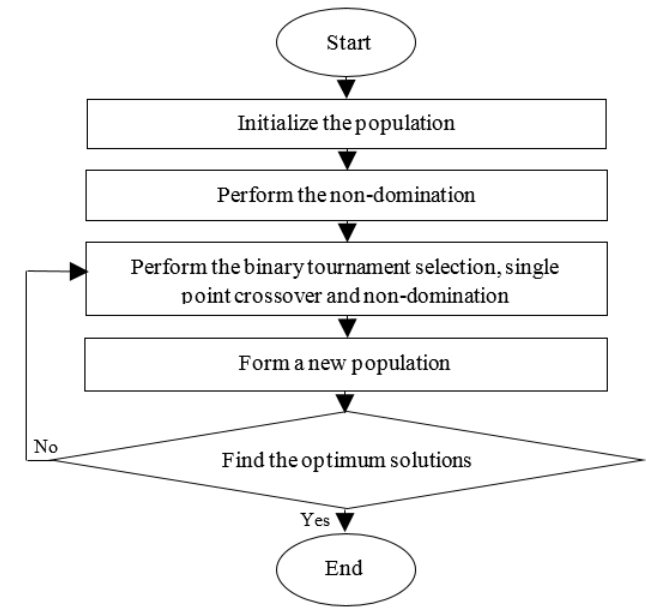

Figure 4. The flowchart of NSGA $\boldsymbol{\Pi}$

For implementing the proposed model in Anylogic software, the agents are defined to the software. After defining the streets and intersections of the study area in Anylogic, the AMWNVD allocation was specified for each emergency center in MATLAB which is imported to Anylogic software. The constraint Voronoi diagram regions are specified for each emergency center in Anylogic as shown in Figure 5. In each Voronoi diagram, two injuries are located. Each injury sent his location and symptoms. Based on their locations, the proper emergency center assigned to them. The structure of delivering the injuries orders to emergency centers is implemented by Statecharts in Anylogic software. Also, NSGA $\boldsymbol{\Pi}$ wayfinding of ambulances is implemented by elements of traffic library in Anylogic. Figure 6 and 7 show the implementation of the proposed multi-agent simulation for the Voronoi dagram of $\mathrm{EC}_{2}$ and $\mathrm{EC}_{1}$ in Anylogic.

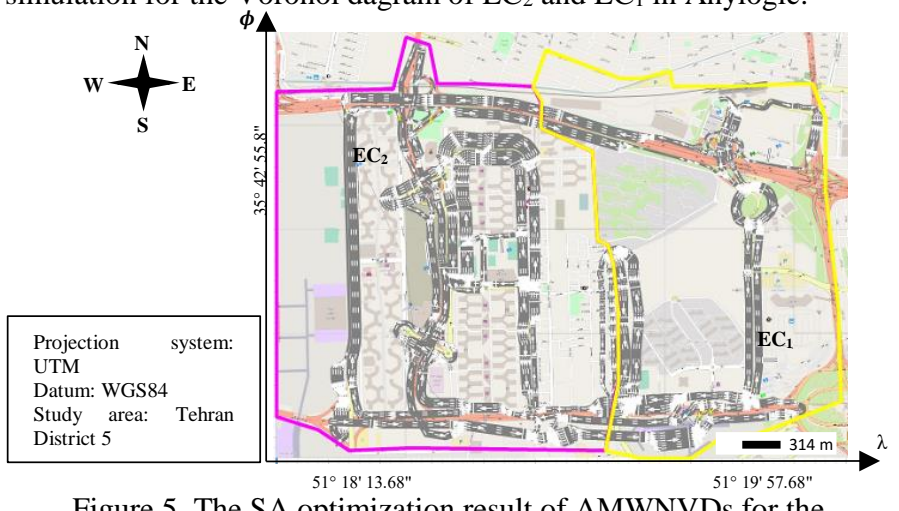

Figure 5. The SA optimization result of AMWNVDs for the emergency centers in Anylogic (the AMWNVD for $\mathrm{EC}_{1}$ and $\mathrm{EC}_{2}$ is shown in yellow and purple, respectively) 


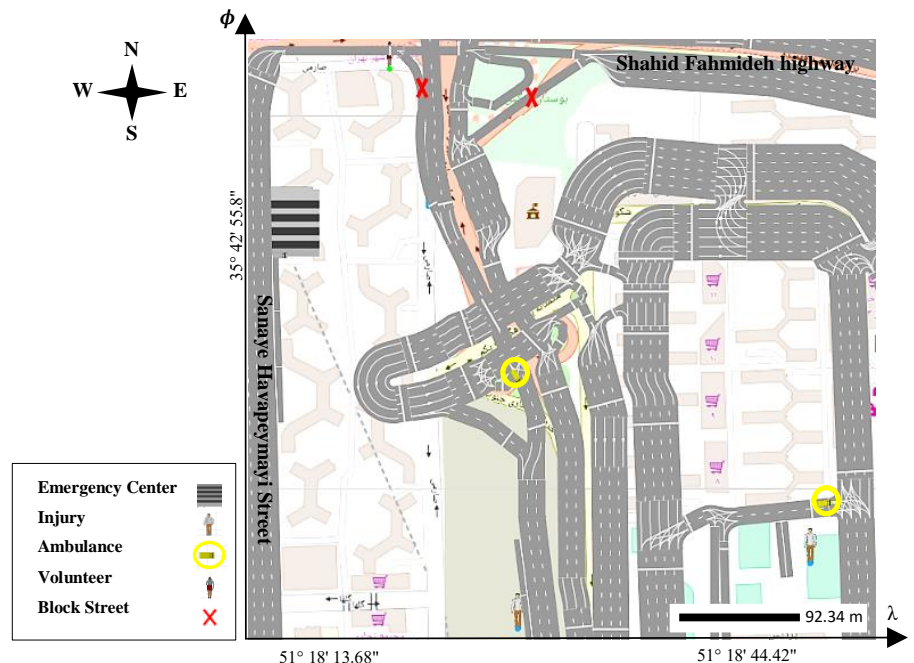

Figure 6. Implementation of the proposed multi-agent simulation for $\mathrm{EC}_{2}$ Voronoi dagram in Anylogic

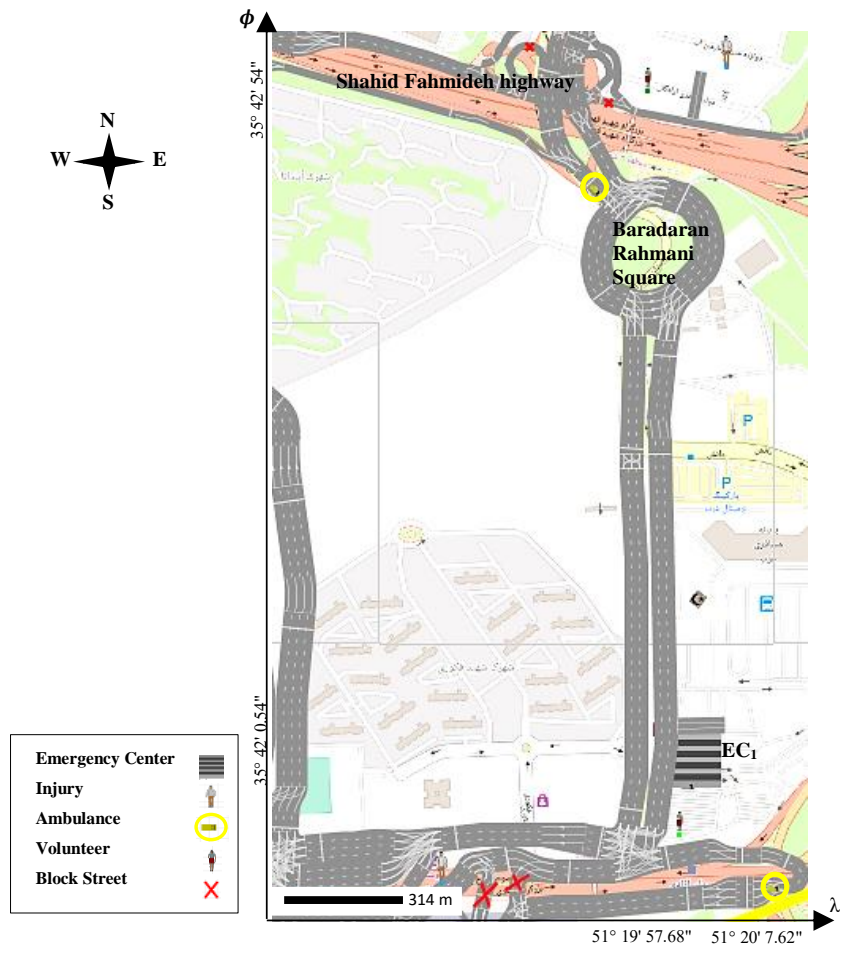

Figure 7. Implementation of proposed multi agent simulation for $\mathrm{EC}_{1}$ Voronoi dagram in Anylogic

In each Voronoi diagram, the integer number is considered as injury agent priority. Table 1 consists of information of the injured agents and their priorities. In the Voronoi diagram of $\mathrm{EC}_{1}$, an ambulance from $\mathrm{EC}_{1}$ is dispatched to Injury 4 location then another ambulance is sent to Injury 1 location based on the injuries priorities. The same process is done in the Voronoi diagram of $\mathrm{EC}_{2}$. The initial time of the ambulances routing and distance of these routes are computed by NSGA $\Pi$, are represented in Table 2 . The volunteers' agents, allocated to their nearest intersection, send the blocked streets information to emergency centers at 3 minutes intervals in Anylogic. So, Wayfinding of the ambulances is updated based on this information at 3 minutes intervals. In this study, the blocked streets are selected randomly. At the first 3 minutes, the streets with id 36, 25 and 91 are reported as the blocked streets. At the second 3 minutes, the streets with id 36, 25 and 91, at the third 3 minutes, the streets with id 36,10189 and 25, at the fourth 3 minutes, the same streets as the third step and at the fifth 3 minutes, the streets with id 10170,10189 and 91 are reported as the blocked streets. At each step, the ambulance route consisting of at least one of the blocked streets is updated by NSGA $\boldsymbol{\Pi}$. After the mentioned five steps, the results of the updated routes are presented in Table 3. If ambulances do not update their routes, get stuck in the blocked streets and the rescue time increases so there would be some threats to the injuries. When the route of the ambulance from $\mathrm{EC}_{1}$ to Injury $1, \mathrm{EC}_{2}$ to Injury $2, \mathrm{EC}_{2}$ to Injury 3 and Injury 4 to $\mathrm{EC}_{1}$ are not updated, the respond time of the ambulances is increased to $16.814,14.368,16.721$ and 18.088 Minutes respectively.

\begin{tabular}{|l|c|c|c|}
\hline Injury & $\begin{array}{c}\text { Emergency } \\
\text { center }\end{array}$ & Priority & Address \\
\hline Injury 1 & EC1 & 3 & Lashkari Expy way \\
\hline Injury 2 & EC2 & 1 & Sattari Expy way \\
\hline Injury 3 & EC1 & 4 & Rahahan St. \\
\hline Injury 4 & EC2 & 5 & Azadegan St. \\
\hline
\end{tabular}

Table 1. Details of the injuries in the study area

\begin{tabular}{|l|c|c|c|}
\hline Origin & Destination & $\begin{array}{c}\text { Distance } \\
\text { (Meters) }\end{array}$ & $\begin{array}{c}\text { Travel time } \\
\text { (Minutes) }\end{array}$ \\
\hline $\mathrm{EC}_{1}$ & Injury 1 & 5187.5720 & 10.694 \\
\hline $\mathrm{EC}_{2}$ & Injury 2 & 3411.011 & 4.522 \\
\hline $\mathrm{EC}_{2}$ & Injury 3 & 4488.802 & 6.874 \\
\hline $\mathrm{EC}_{1}$ & Injury 4 & 3204.855 & 9.277 \\
\hline Injury 1 & $\mathrm{EC}_{1}$ & 3713.9890 & 6.702 \\
\hline Injury 2 & $\mathrm{EC}_{2}$ & 5211.755 & 8.840 \\
\hline Injury 3 & $\mathrm{EC}_{2}$ & 4395.162 & 5.028 \\
\hline Injury 4 & $\mathrm{EC}_{1}$ & 3135.266 & 4.829 \\
\hline
\end{tabular}

Table 2. Details of initial wayfinding ambulances from the emergency center to the injury location and vice versa

So, comparing the travel time of the ambulances when get stuck in traffic with results of Table 3 shows the rescue time decreased about in $36.7 \%$ in the proposed model.

\begin{tabular}{|l|c|c|c|}
\hline Origin & Destination & $\begin{array}{c}\text { Distance } \\
\text { (Meters) }\end{array}$ & $\begin{array}{c}\text { Travel time } \\
\text { (Minutes) }\end{array}$ \\
\hline $\mathrm{EC}_{1}$ & Injury1 & 6497.1845 & 12.568 \\
\hline $\mathrm{EC}_{2}$ & Injury2 & 4600.081 & 12.346 \\
\hline $\mathrm{EC}_{2}$ & Injury3 & 5700.073 & 11.032 \\
\hline Injury4 & $\mathrm{EC}_{1}$ & 3175.4255 & 4.865 \\
\hline
\end{tabular}

Table 3. Details of updated ambulances routes during five steps

In this method, the time required to get the wounded medical assistance after the occurrence of a natural disaster is decreased. The results show this method accelerates the allocation of the ambulances to the wounded and wayfinding of the ambulances in the rescue operation after a natural disaster in multi-agent simulation. The rescue time depends on the distinguishing blocked street in real time and avoiding from these streets in the rescue operation. So, the injuries receive assistance on time and the casualties of disaster decreases.

\section{CONCLUSION}

In this paper, the multi-agent rescue operation that integrated the optimal allocation of the ambulances to the wounded locations and the wayfinding of the ambulances based on facilities of the smart city to expedite the rescue operation after a natural disaster, have been proposed. The proposed multi-agent system consisting of emergency centers, streets, injuries, ambulances, volunteers 
and their rescue schedule agents. In this system, the constrained Voronoi diagrams were used for space allocation of urban emergency centers according to the population, supply and street network. The number of ambulances in each emergency center considered as emergency centers weight.

Simulated annealing optimization algorithm is used for minimizing the difference between demands and supplies of the constrained network Voronoi diagrams. NSGA $\Pi$ was considered as a multi objective routing algorithm for assigning emergency services to the injuries and transferring them to the emergency centers that minimized the ambulance travel time and route length. Also, this system is implemented in Anylogic simulation software. A part of Municipal District 5 of Tehran is considered as a study area. In this system, the location of the wounded agents and the symptom of the injuries are sent to the emergency centers. After determining the constraint Voronoi diagram of the wounded person, the emergency center that is located in this Voronoi diagram sends the ambulance to the wounded person location. The NSGA $\boldsymbol{\Pi}$ algorithm is used for finding the optimum path of the dispatched ambulances. This process was performed simultaneously for multi injuries in different Voronoi diagrams. The severity of the injury victims determines the priorities of the injuries for receiving medical assistance. In this paper, for specifying the blocked streets in real time, volunteered residents as sensors reported impassable streets during 3 minutes intervals. Wayfinding of the ambulances is updated based on this information at 3 minutes intervals. The new optimum paths of the ambulances routing transfer to ambulances. Using this strategy, the proposed system would be closer to real time. The difference between the supply and the demand divided to the supply in each Voronoi diagram decreased to 0.1601 and this method decreased the response time of the ambulances about in $36.7 \%$.

Our future work will be focused on testing the proposed model for consideration of the real-time traffic condition of the streets recorded by the sensors, implementation of the smart transportation for wayfinding of the ambulances and participating more volunteers for reporting the blocked streets and the wounded location.

\section{REFERENCES}

Aurenhammer, F., 1991. Voronoi diagrams - a survey of a fundamental geometric data structure. ACM Computing Surveys (CSUR), 23, pp. 345-405.

Bakıc1, T., E. Almirall and J. Wareham., 2013. A smart city initiative: the case of Barcelona. Journal of the Knowledge Economy, 4, pp. 135-148.

Chavoshi, S. H., M. R. Delavar, M. R. Malek and A. Frank., 2008. Landmark-Based Simulation for Agent Wayfinding after Earthquake.

Deb, K., A. Pratap, S. Agarwal and T. Meyarivan., 2002. A fast and elitist multiobjective genetic algorithm: NSGA-II. IEEE transactions on evolutionary computation, 6, pp. 182-197.

Foresti, G. L., M. Farinosi and M. Vernier., 2015. Advanced Emergency Response Management in Smart Environments. In Encyclopedia of Information Science and Technology, Third Edition, pp. 13371349. IGI Global.

Graf, M. and S. Winter. 2003. Network-voronoi-diagram. Österreichische Zeitschrift für Vermessung und Geoinformation, 91, pp. 166-174.

Hirokawa, N. and T. Osaragi., 2016. Access Time of Emergency Vehicles Under the Condition of Street Blockages after a Large Earthquake. ISPRS-International Archives of the Photogrammetry, Remote Sensing and Spatial Information Sciences, pp. 37-44.

Horita, F. E. A., L. C. Degrossi, L. F. G. de Assis, A. Zipf and J. P. de Albuquerque., 2013. The use of volunteered geographic information (VGI) and crowdsourcing in disaster management: a systematic literature review.

Karimi F., 2009. Optimization of Urban facilities Space Allocation Using Constrained Voronoi Diagrams, In School of Surveying and Geospatial Engineering. college of engineering: MSC theisis. University of Tehran.

Li, X., Z. Zhao, X. Zhu and T. Wyatt., 2011. Covering models and optimization techniques for emergency response facility location and planning: a review. Mathematical Methods of Operations Research, 74, pp. 281-310.

Macal, C. M. and M. J. North., 2005. Tutorial on agent-based modeling and simulation. In Simulation Conference, 2005 Proceedings of the Winter, 14 pp.: IEEE.

Mitchell, M., 1998. An introduction to Genetic Algorithms. MIT press.

Okabe, A., B. Boots and K. Sugihara., 2000. Spatial tessellations: concepts and applications of Voronoi diagrams, the second edition, pp. 362-363.

Okabe, A., T. Satoh, T. Furuta, A. Suzuki and K. Okano., 2008. Generalized network Voronoi diagrams: Concepts, computational methods, and applications. International Journal of Geographical Information Science, 22, pp. 965-994.

Poblet, M., E. García-Cuesta and P. Casanovas., 2017. Crowdsourcing roles, methods and tools for dataintensive disaster management. Information Systems Frontiers, pp. 1-17.

Reitsma, R., S. Trubin and E. Mortensen., 2007. Weightproportional space partitioning using adaptive Voronoi diagrams. Geoinformatica, 11, 383-405.

Reitsma, R., S. Trubin and S. Sethia. 2004. Information space regionalization using adaptive multiplicatively weighted Voronoi diagrams. In Information Visualisation, 2004. IEEE. Eighth International Conference on, pp. 290-294

Roozemond ,D. A., 2001. Using intelligent agents for pro-active, real-time urban intersection control. European Journal of Operational Research, 131, pp. 293-301.

Rusell, S. and P. Norvig., 2003. Artificial Intelligent: A modern approach.

Seshadri, A., 2007. A fast elitist multiobjective genetic algorithm: NSGA-II, Mathlab Central, file exchange, mathworks.

Srinivas, N. and K. Deb., 1994. Muiltiobjective optimization using nondominated sorting in genetic algorithms. Evolutionary computation, 2, pp. 221-248.

Wang, Z. and S. Zlatanova., 2016. Multi-agent based path planning for first responders among moving obstacles. Computers, Environment and Urban Systems, 56, pp.

Yang, X.-S., 2010. Engineering optimization: an introduction with metaheuristic applications. John Wiley and Sons. 